\title{
Multiple Grid Methods for the Solution of Fredholm Integral Equations of the Second Kind
}

\author{
By P. W. Hemker and H. Schippers
}

\begin{abstract}
In this paper multiple grid methods are applied for the fast solution of the large nonsparse systems of equations that arise from the discretization of Fredholm integral equations of the second kind. Various multiple grid schemes, both with Nyström and with direct interpolation, are considered. For these iterative methods, the rates of convergence are derived using the collectively compact operator theory by Anselone and Atkinson. Estimates for the asymptotic computational complexity are given, which show that the multiple grid schemes result in $\theta\left(N^{2}\right)$ arithmetic operations.
\end{abstract}

1. Introduction. Multiple grid methods have been advocated by Brandt [5], [6] for solving sparse systems of equations that arise from discretization of partial differential equations. Convergence and computational complexity of such multiple grid techniques have been studied since by Hackbusch [7], [8] and Wesseling [12], [13]. We intend to show that multiple grid methods can also be used advantageously for the nonsparse systems that occur in numerical methods for integral equations.

In a recent paper [10], the second author applied the multiple grid technique to the solution of Fredholm integral equations of the second kind

$$
f(x)-\int_{0}^{1} k(x, y) f(y) d y=g(x), \quad x \in[0,1],
$$

where $g$ belongs to a Banach space $X$. At the same time, Hackbusch [7] also used a multiple grid technique for these problems. Moreover, he gave a proof of convergence. In this proof he assumed the operator $K$, associated with the kernel $k(x, y)$, to map from $X$ into a "smooth" subspace $\tilde{X} \subset X$, which has a stronger topology. In the present paper, for Hackbusch's method we give another proof, which fits into the theoretical framework developed by Anselone [1] and Atkinson [2], [3] for Fredholm equations. We assume that $K$ is compact from $X$ into $X$. In contrast to Hackbusch's analysis, this approach enables us to consider also Nyström interpolation as a permissible interpolation method. In addition, we introduce a new multiple grid method for Fredholm integral equations, which can deal with a larger class of problems than the method proposed by Hackbusch.

In 1978 Stetter [11] introduced the Defect Correction Principle for the formulation of various iterative methods. We shall apply this principle because it also appears to be an expedient tool to formulate multiple grid techniques.

Received January 17, 1980.

1980 Mathematics Subject Classification. Primary 65B99, 65F10, 65R20.

Key words and phrases. Fredholm integral equations of the second kind, multiple grid methods. 
In Section 2 we collect some results from papers by Atkinson [2], [3] and Prenter [9]. In Section 3 we cast the iterative schemes of Brakhage [4] and Atkinson [2], [3] into the context of the Defect Correction Principle and multiple grid iteration. Furthermore, we give the proof of convergence of the multiple grid schemes with Nyström interpolation. In Section 4 we treat other interpolation methods and we extend the iterative schemes of Section 3 for subspaces $X_{p}$ of $X$ of finite dimension $N_{p}$. These schemes are used as a basis for the construction of a general algorithm for the solution of Fredholm equations of the second kind. This algorithm is more efficient than the algorithms by Brakhage [4] and Atkinson [2], [3] because these schemes take $\vartheta\left(N_{p}^{3}\right)$ and $\vartheta\left(N_{p}^{2} \log N_{p}\right)$ operations, respectively, whereas the multiple grid schemes result in $\Theta\left(N_{p}^{2}\right)$ operations. In Section 5 we illustrate the theoretical results of the previous sections by some numerical examples and we comment on the computational complexity.

2. Basic Assumptions. Equation (1.1) can be written symbolically as

$$
A f=g, \quad g \in X,
$$

where $X$ is a Banach space and $A=I-K$, with $I$ the identity operator on $X$ and $K$ the linear operator associated with the kernel $k(x, y) . A$ is assumed to have a bounded inverse on $X$. We shall discuss the convergence of a sequence of approximations to the unique solution of (2.1).

Let $X_{p}, p=0,1,2, \ldots$, be finite-dimensional subspaces of $X$ and let $T_{p}$, $p=0,1,2, \ldots$, be a bounded projection operator from $X$ onto $X_{p}$, i.e. $T_{p} f=f$ for all $f \in X_{p}$. We need the following assumptions for $\left\{X_{p}\right\}$ and $\left\{T_{p}\right\}$

(A1) $X_{0} \subset X_{1} \subset \cdots \subset X_{p} \subset \cdots \subset X$,

(A2) $\lim _{p \rightarrow \infty}\left\|f-T_{p} f\right\|=0$ for all $f \in X$.

LEMMA 2.1.

$$
C_{1}=\sup _{p>0}\left\|T_{p}\right\|<\infty
$$

Proof. The lemma follows from the principle of uniform boundedness; see Atkinson [3, p. 18].

The sequence $\left\{X_{p}\right\}$ is thought to be associated with a sequence of decreasing meshsizes $\left\{h_{p}\right\}$ with $\lim _{p \rightarrow \infty} h_{p}=0$. Corresponding with this sequence $\left\{h_{p}\right\}$, we approximate $K$ by a sequence of operators $\left\{K_{p}\right\}, K_{p}: X \rightarrow X$. Analogous to $A=I-K$, we also write $A_{p}=I-K_{p}$. In the context of multiple grid iteration, the subscript $p$ is called "level".

We use the following assumptions on $K_{p}, p=0,1,2, \ldots$,

(A3) $K_{p}$ is a linear operator $X \rightarrow X$.

(A4) $\left\{K_{p}\right\}$ is a collectively compact family of operators.

(A5) $\lim _{p \rightarrow \infty}\left\|K_{p} f-K f\right\|=0$ for all $f \in X$.

(A6) $K_{p}=K_{p} T_{p}$.

LEMMA 2.2. From the assumptions (A3)-(A5) follow:

(i) $K$ should be compact.

(ii) The sequence $\left\{K_{p}\right\}$ is uniformly bounded, i.e.

$$
C_{2}=\sup _{p>0}\left\|K_{p}\right\|<\infty \text {. }
$$


(iii) $\lim _{p \rightarrow \infty}\left\|\left(K-K_{p}\right) M\right\|=0$ for any compact operator $M: X \rightarrow X$.

(iv) Let

$$
a_{p}=\sup _{q>p} \sup _{l>0}\left\|\left(K-K_{q}\right) K_{l}\right\|
$$

Then $\lim _{p \rightarrow \infty} a_{p}=0$.

Proof. See Atkinson [3, pp. 96 and 138].

LEMMA 2.3. Let the finite-dimensional subspace $X_{0} \subset X$ be sufficiently large (i.e. the meshwidth of the coarsest discretization is sufficiently small). From the existence of a bounded inverse of $A=I-K$ and the assumptions (A3)-(A5) follow:

(i) $\left(I-K_{p}\right)^{-1}$ exists on $X$ for $p \geqslant 0$ and

$$
C_{3}=\sup _{p>0}\left\|\left(I-K_{p}\right)^{-1}\right\|<\infty .
$$

(ii) $\left\|f-f_{p}\right\| \leqslant C_{3}\left\|K f-K_{p} f\right\|$, where $f$ is the solution of (2.1) and $f_{p}$ of

$$
\left(I-K_{p}\right) f_{p}=g \text {. }
$$

Proof. See Atkinson [2, p. 18].

The following lemma is a summary of results given by Prenter [9].

LEMMA 2.4. From the assumptions (A2)-(A6) follow:

(i) For any compact operator $M$ on $X$ into $X$,

$$
\lim _{p \rightarrow \infty}\left\|\left(I-T_{p}\right) M\right\|=0 .
$$

(ii) If $X_{0}$ is sufficiently large, then $\left(I-T_{p} K_{p}\right)^{-1}$ exists on $X$ for $p \geq 0$ and

$$
C_{4}=\sup _{p>0}\left\|\left(I-T_{p} K_{p}\right)^{-1}\right\|<\infty .
$$

Let $\tilde{f}_{p}$ be a solution of

$$
\left(I-T_{p} K_{p}\right) \tilde{f}_{p}=T_{p} g .
$$

According to Lemma 2.4(ii), $\tilde{f}_{p}$ exists and is unique; it follows from (2.3) that $\tilde{f}_{p} \in X_{p}$.

LEMMA 2.5. Let

$$
b_{p}=\sup _{q>p} \sup _{l>0}\left\|\left(I-T_{q}\right) K_{l}\right\|
$$

Then $\lim _{p \rightarrow \infty} b_{p}=0$.

Proof. Let $\Psi=\left\{K_{p} f \mid p \geqslant 0\right.$ and $\left.\|f\|<1\right\}$. By assumption (A4), $\Psi$ has compact closure in the Banach space $X$. Then

$$
b_{p}=\sup _{q>p} \sup _{z \in \Psi}\left\|\left(I-T_{q}\right) z\right\|
$$

and the proof follows by assumption (A2).

Lemma 2.6. Let the subspace $X_{0} \subset X$ be sufficiently large. Then

$$
\left\|\tilde{f}_{p}-T_{p} f\right\| \leqslant C_{1} C_{4}\left\|K f-K_{p} f\right\|
$$

where $f$ is the solution of (2.1). 
Proof. See Prenter [9, Theorem 6.3].

As a consequence of assumption (A1), the following lemma is trivial.

LeMma 2.7. Let $q \leqslant p$, i.e. $\operatorname{dim}\left(X_{q}\right) \leqslant \operatorname{dim}\left(X_{p}\right)$. Then

$$
T_{p} T_{q}=T_{q} T_{p}=T_{q}
$$

3. Iteration Schemes With Nyström Interpolation. In this section we use the Defect Correction Principle (cf. Stetter [11]) to formulate a class of iterative methods for the solution of (2.2). This equation is written as

$$
A_{p} f_{p}=g, \quad g \in X,
$$

with $A_{p}=I-K_{p}$. The defect correction principle defines the following iterative process:

$$
\left\{\begin{array}{l}
f_{p, 0}=0 \\
f_{p, i+1}=B_{p} g+\left(I-B_{p} A_{p}\right) f_{p, i} .
\end{array}\right.
$$

Here $B_{p}$ denotes some approximate inverse of $A_{p}$, which is bijective and continuous in $X$. The solution $f_{p}$ of (3.1) is a fixed point of (3.2), and (3.2) will converge to $f_{p}$ if the rate of convergence $\left\|I-B_{p} A_{p}\right\|<1$.

Several well-known iterative schemes for solving Fredholm integral equations of the second kind can be formulated within this framework. The iterative scheme of Brakhage [4] is obtained by taking the following approximate inverse

$$
B_{p}^{(1)}=I+\left(I-K_{p-1}\right)^{-1} K_{p} \text {. }
$$

Here we notice that the operator $\left(I-K_{q}\right)^{-1}, q \geqslant 0$, as a mapping on $X$ into $X$ describes the process of discretization, solution of the discrete problem (i.e. the solution of a square linear system), and subsequent Nyström interpolation; see e.g. [10]. Other kinds of interpolation are treated in the next section.

The second iterative scheme of Atkinson [2, p. 19], arises from

$$
B_{p}^{(2)}=I+\left(I-K_{0}\right)^{-1} K_{p} \text {. }
$$

The rates of convergence of the corresponding iterative processes are estimated in the following theorem.

THEOREM 3.1. (i) $\left\|I-B_{p}^{(1)} A_{p}\right\| \rightarrow 0$ as $p \rightarrow \infty$,

(ii) $\left\|I-B_{p}^{(2)} A_{p}\right\| \leqslant C\left(X_{0}\right)$ as $p \rightarrow \infty, C\left(X_{0}\right)<1$ for $X_{0}$ sufficiently large.

Proof. (i) Substitution of the explicit expressions for $A_{p}$ and $B_{p}^{(1)}$ yields

$$
\begin{aligned}
I-B_{p}^{(1)} A_{p} & =I-\left\{I+\left(I-K_{p-1}\right)^{-1} K_{p}\right\}\left(I-K_{p}\right) \\
& =K_{p}-\left(I-K_{p-1}\right)^{-1} K_{p}\left(I-K_{p}\right)=\left(I-K_{p-1}\right)^{-1}\left(K_{p}-K_{p-1}\right) K_{p} .
\end{aligned}
$$

From Lemmas 2.2 and 2.3, we get the following bound for the norm

$$
\left\|\left(I-K_{p-1}\right)^{-1}\left(K_{p}-K_{p-1}\right) K_{p}\right\| \leqslant C_{3}\left(a_{p}+a_{p-1}\right) .
$$

(ii) Analogously, we get, for $B_{p}^{(2)}$ with $X_{0}$ sufficiently large,

$$
\left\|I-B_{p}^{(2)} A_{p}\right\| \leqslant C_{3}\left(a_{p}+a_{0}\right) \equiv C\left(X_{0}\right) .
$$

From Lemma 2.2 it follows that $C\left(X_{0}\right)<1$ for all sufficiently large $X_{0}$. 
We remark that the approximate inverses $B_{p}^{(1)}$ and $B_{p}^{(2)}$ use only two levels: $B_{p}^{(1)}$ uses the levels $p-1$ and $p$, whereas $B_{p}^{(2)}$ uses the levels 0 and $p$. We now introduce approximate inverses $B_{p}^{(3)}$ and $B_{p}^{(4)}$, which use $p+1$ levels. They are defined recursively as follows:

$$
\left\{\begin{array}{l}
B_{0}^{(3)}=\left(I-K_{0}\right)^{-1}, \\
B_{p}^{(3)}=I+Q_{p-1}^{(3)} K_{p}, \quad p=1,2, \ldots,
\end{array}\right.
$$

and

$$
\left\{\begin{array}{l}
B_{0}^{(4)}=\left(I-K_{0}\right)^{-1}, \\
B_{p}^{(4)}=Q_{p-1}^{(4)}\left(I-K_{p-1}+K_{p}\right), \quad p=1,2, \ldots,
\end{array}\right.
$$

with $Q_{p}^{(j)}, j=3,4, p=0,1,2, \ldots$, given by

$$
Q_{p}^{(j)}=\sum_{m=0}^{\gamma-1}\left(I-B_{p}^{(j)} A_{p}\right)^{m} B_{p}^{(j)}
$$

for some positive integer $\gamma$.

From the fact that $Q_{p}^{(j)}$ satisfies the equality

$$
\left(I-Q_{p}^{(j)} A_{p}\right)=\left(I-B_{p}^{(j)} A_{p}\right)^{\gamma}
$$

we see that $Q_{p}^{(j)}$ is an approximate inverse of $A_{p}$ and its application is equivalent with the application of $\gamma$ iteration steps of (3.2) with the use of the approximate inverse $B_{p}^{(j)}$. In fact, this is the motivation for this definition of $Q_{p}^{(j)}$ and it is the basis for the actual (recursive) implementation of the method.

In the following definition we give a short notation for the rates of convergence for the various iterative processes.

Definition.

$$
\zeta_{p}^{(j)}=\left\|I-B_{p}^{(j)} A_{p}\right\|, \quad j=1,2,3,4
$$

THEOREM 3.2. (i) $\zeta_{p}^{(3)} \leqslant \zeta_{p}^{(1)}+\zeta_{p-1}^{(3)^{\gamma}}\left(\zeta_{p}^{(1)}+\left\|K_{p}\right\|\right)$,

(ii) $\zeta_{p}^{(4)} \leqslant \zeta_{p}^{(1)}+\zeta_{p-1}^{(4)}\left(\zeta_{p}^{(1)}+1\right)$.

Proof. (i) By definition

$$
I-B_{p}^{(1)} A_{p}=I-\left\{I+A_{p-1}^{-1}\left(I-A_{p}\right)\right\} A_{p}
$$

and

$$
\begin{aligned}
I-B_{p}^{(3)} A_{p} & =I-\left\{I+\left[I-\left(I-B_{p-1}^{(3)} A_{p-1}\right)^{\gamma}\right] A_{p-1}^{-1}\left(I-A_{p}\right)\right\} A_{p} \\
& =I-B_{p}^{(1)} A_{p}+\left(I-B_{p-1}^{(3)} A_{p-1}\right)^{\gamma} A_{p-1}^{-1}\left(I-A_{p}\right) A_{p} \\
& =I-B_{p}^{(1)} A_{p}+\left(I-B_{p-1}^{(3)} A_{p-1}\right)^{\gamma}\left(B_{p}^{(1)}-I\right) A_{p} .
\end{aligned}
$$

Hence,

$$
\left\|I-B_{p}^{(3)} A_{p}\right\| \leqslant\left\|I-B_{p}^{(1)} A_{p}\right\|+\left\|I-B_{p-1}^{(3)} A_{p-1}\right\|^{\gamma}\left\{\left\|I-B_{p}^{(1)} A_{p}\right\|+\left\|I-A_{p}\right\|\right\},
$$

i.e.

$$
\zeta_{p}^{(3)} \leqslant \zeta_{p}^{(1)}+\zeta_{p-1}^{(3)}\left(\zeta_{p}^{(1)}+\left\|K_{p}\right\|\right)
$$


(ii) Similar to the case (i), now we have

$$
\begin{aligned}
I-B_{p}^{(4)} A_{p} & =I-\left[I-\left(I-B_{p-1}^{(4)} A_{p-1}\right)^{\gamma}\right] A_{p-1}^{-1}\left(A_{p-1}-A_{p}+I\right) A_{p} \\
& =I-B_{p}^{(1)} A_{p}+\left(I-B_{p-1}^{(4)} A_{p-1}\right)^{\gamma} B_{p}^{(1)} A_{p} .
\end{aligned}
$$

Hence, i.e.

$$
\left\|I-B_{p}^{(4)} A_{p}\right\| \leqslant\left\|I-B_{p}^{(1)} A_{p}\right\|+\left\|I-B_{p-1}^{(4)} A_{p-1}\right\|^{\gamma}\left\|I-B_{p}^{(1)} A_{p}-I\right\|,
$$

$$
\zeta_{p}^{(4)} \leqslant \zeta_{p}^{(1)}+\zeta_{p-1}^{(4)^{\gamma}}\left(\zeta_{p}^{(1)}+1\right) .
$$

By Theorem 3.1 we know that $\zeta_{p}^{(1)} \rightarrow 0$ as $p \rightarrow \infty$; conditions for $\zeta_{p}^{(3)}$ to vanish depend on $\gamma,\left\|K_{p}\right\|$, and $\zeta_{p}^{(1)}$, whereas the conditions for $\zeta_{p}^{(4)}$ depend on $\gamma$ and $\zeta_{p}^{(1)}$ only. In order to study this dependence further, we prove the following lemma.

Lemma 3.3. Let $k \in \mathbf{R}$ and $\gamma \in \mathbf{N}$ be given, let $\left\{v_{p} \mid v_{p}>0, p=0,1,2, \ldots\right\}$ be a nonincreasing sequence with $d=\inf _{p} v_{p} / v_{p-1}$ and let $\left\{w_{p}\right\}$ be defined by

$$
\left\{\begin{array}{l}
w_{0}=v_{0} \\
w_{p}=v_{p}+w_{p-1}^{\gamma}\left(v_{p}+k\right) .
\end{array}\right.
$$

If either

$$
\left\{\begin{array}{l}
\gamma \geqslant 1, \quad 0<k<d<1, \quad \text { and } \\
v_{0}<\frac{1}{2}(1-k / d),
\end{array}\right.
$$

or

$$
\left\{\begin{array}{l}
\gamma \geqslant 2, \quad \text { and } \\
v_{0}<\frac{1}{2}\left\{\sqrt{1+(k / d)^{2}}-k / d\right\}
\end{array}\right.
$$

then a $C>0$ exists such that $v_{p} \leqslant w_{p} \leqslant C v_{p}$.

Proof. (i) We define $c=(d+k) /(d-k)$. Then $c>1$ and the conditions on $\left\{v_{p}\right\}$ are written as

$$
k \frac{c+1}{c-1}<\frac{v_{p}}{v_{p-1}} \leqslant 1
$$

and

$$
(1+c) v_{0}<1
$$

We show that the lemma is true for $C=1+c$. From (3.8) we see $w_{0}=v_{0}<$ $(1+c) v_{0}<1$. Now we show by induction that $w_{p}<(1+c) v_{p}<1$ assuming that $w_{p-1}<(1+c) v_{p-1}<1$.

From (3.7) follows

$$
\begin{aligned}
& \frac{v_{p-1}}{v_{p}} k<\frac{c-1}{c+1}, \\
& \left(\frac{1}{c+1}+\frac{v_{p-1}}{v_{p}} k\right)<\frac{c}{c+1}, \\
& (1+c)^{\gamma-1} v_{p-1}^{\gamma-1}\left(v_{p-1}+\frac{v_{p-1}}{v_{p}} k\right)<\frac{c}{c+1}, \\
& w_{p-1}^{\gamma}\left(v_{p}+k\right)<(1+c)^{\gamma} v_{p-1}^{\gamma}\left(v_{p}+k\right)<c v_{p}, \\
& w_{p}=v_{p}+w_{p-1}^{\gamma}\left(v_{p}+k\right)<(1+c) v_{p} .
\end{aligned}
$$


(ii) We assume $v_{0}<\sqrt{(k / 2 d)^{2}+c /(1+c)^{2}}-k / 2 d$ for some $0<c<1$ and we show $w_{p} \leqslant(1+c) v_{p}$. Then the lemma is proven by taking $c=1$.

For any $v \in\left[0, v_{0}\right]$, we have

$$
v^{2}+\frac{k}{d} v-\frac{c}{(1+c)^{2}}<0
$$

Hence, $(1+c) v(v+k / d)<c /(1+c)$. By assumption, we know

$$
w_{0}=v_{0}<(1+c) v_{0}<\sqrt{\left\{\frac{k(1+c)}{2 d}\right\}^{2}+c}-\frac{k(1+c)}{2 d}<\sqrt{c}<1 .
$$

Now we show by induction that $w_{p}<(1+c) v_{p}<1$, assuming that $w_{p-1}<$ $(1+c) v_{p-1}<1$,

$$
\begin{aligned}
& (1+c) v_{p-1}\left(v_{p-1}+\frac{k}{d}\right)<\frac{c}{1+c}, \\
& (1+c) v_{p-1}\left(v_{p-1}+\frac{v_{p-1}}{v_{p}} k\right)<\frac{c}{1+c}, \\
& (1+c)^{2} v_{p-1}^{2}\left(1+\frac{k}{v_{p}}\right)<c, \\
& (1+c)^{\gamma} v_{p-1}^{\gamma}\left(1+\frac{k}{v_{p}}\right)<c, \quad \gamma=2,3, \ldots, \\
& w_{p-1}^{\gamma}\left(1+\frac{k}{v_{p}}\right)<c, \\
& w_{p}=v_{p}+w_{p-1}^{\gamma}\left(v_{p}+k\right)<(1+c) v_{p} . \text { Q.E.D. }
\end{aligned}
$$

THEOREM 3.4. Let $\gamma \geqslant 2$ and let $\zeta_{p}^{(1)}$ satisfy

$$
\zeta_{p}^{(1)} \leqslant v_{p}=d^{p} v_{0} \text { for some } 0<d<1 .
$$

Then,

(i) if $v_{0} \leqslant\left\{\sqrt{d^{2}+C_{2}^{2}}-C_{2}\right\} / 2 d$, it follows that $\zeta_{p}^{(3)} \leqslant 2 d^{p} v_{0}$, and

(ii) if $v_{0} \leqslant\left\{\sqrt{1+d^{2}}-1\right\} / 2 d$, it follows that $\zeta_{p}^{(4)}<2 d^{p} v_{0}$.

Proof. (i) Let $\left\{w_{p}\right\}$ be defined as in Lemma 3.3 with $k=C_{2}=\sup _{p>0}\left\|K_{p}\right\|$. Then it follows from the proof of Lemma 3.3 that $w_{p} \leqslant 2 v_{p}$. Therefore we show $\zeta_{p}^{(3)} \leqslant w_{p}$ by induction: from the definition of $\zeta_{p}^{(3)}$ we derive

$$
\zeta_{0}^{(3)}=\zeta_{0}^{(1)} \leqslant v_{0}=w_{0}
$$

and, by Theorem 3.2,

$$
\zeta_{p}^{(3)} \leqslant \zeta_{p}^{(1)}+\zeta_{p-1}^{(3)^{\gamma}}\left(\zeta_{p}^{(1)}+\left\|K_{p}\right\|\right) \leqslant v_{p}+w_{p-1}^{\gamma}\left(v_{p}+C_{2}\right)=w_{p} .
$$

(ii) Similarly, with $\left\{w_{p}\right\}$ defined as in Lemma 3.3 with $k=1$, we prove $\zeta_{p}^{(4)}<w_{p}$ and, hence,

$$
\zeta_{p}^{(4)}<w_{p}<2 v_{p}=2 d^{p} v_{0} \text {. Q.E.D. }
$$


Remark. If $B_{p}^{(3)}$ is defined with $\gamma=1$, then a similar proof yields that, for any decreasing sequence $\left\{v_{p}\right\}$ with

$$
\sup _{p>0}\left\|K_{p}\right\|=C_{2}<d=\inf _{p} \frac{v_{p}}{v_{p-1}}<1
$$

for which $\zeta_{p}^{(1)} \leqslant v_{p}$, we have $\zeta_{p}^{(3)}<\left(2 d /\left(d-C_{2}\right)\right) v_{p}$.

By $f_{p, \sigma}^{(j)}$ we denote the result of $\sigma$ applications of the Defect Correction Process on level $p$ with approximate inverse $B_{p}^{(j)}, j=1,3,4$, when we take zero as the initial approximant.

With the aid of the previous theorem and Lemma 2.3, now the following theorem is immediate.

THEOREM 3.5 [APPROXIMATION THEOREM]. Under the hypotheses of Theorem 3.4, the Multi Grid process yields approximate solutions for which the following error estimates hold

$$
\left\|f-f_{p, \sigma}^{(j)}\right\| \leqslant C_{3}\left\|K_{p} f-K f\right\|+\left(2 d^{p} v_{0}\right)^{\sigma}\left\|f_{p}\right\|, \quad j=3,4,
$$

where $f$ and $f_{p}$ are the solutions of (2.1) and (2.2) respectively.

Proof. The proof follows immediately from

$$
\left\|f-f_{p, \sigma}^{(j)}\right\| \leqslant\left\|f-f_{p}\right\|+\left\|f_{p}-f_{p, \sigma}^{(j)}\right\| .
$$

4. Iteration Schemes With Projection into Finite-Dimensional Subspaces. In this section we expand the technique used in Section 3 to find the solution in $X_{p}$ of the equation (2.3):

$$
\tilde{A}_{p} \tilde{f}_{p}=g_{p}, \quad g_{p} \in X_{p},
$$

where $\tilde{A}_{p}=I-T_{p} K_{p}$ is a mapping on $X$ into $X$. We assume that $X_{0}$ is sufficiently large such that $\left(I-T_{p} K_{p}\right)^{-1}$ exists for all $p \geq 0$.

Analogous to the approximate inverses of $A_{p}$ in the previous section, we now introduce

$$
\begin{aligned}
& \tilde{B}_{p}^{(1)}=T_{p}+T_{p-1}\left(I-T_{p-1} K_{p-1}\right)^{-1} T_{p} K_{p}, \\
& \tilde{B}_{p}^{(2)}=T_{p}+T_{0}\left(I-T_{0} K_{0}\right)^{-1} T_{p} K_{p}, \\
& \left\{\begin{array}{l}
\tilde{B}_{0}^{(3)}=T_{0}\left(I-T_{0} K_{0}\right)^{-1}, \\
\tilde{B}_{p}^{(3)}=T_{p}+T_{p-1} \tilde{Q}_{p-1}^{(3)} T_{p} K_{p},
\end{array}\right. \\
& \left\{\begin{array}{l}
\tilde{B}_{0}^{(4)}=T_{0}\left(I-T_{0} K_{0}\right)^{-1}, \\
\tilde{B}_{p}^{(4)}=T_{p}-T_{p-1}+T_{p-1} \tilde{Q}_{p-1}^{(4)}\left(T_{p-1}-T_{p-1} K_{p-1}+T_{p} K_{p}\right),
\end{array}\right.
\end{aligned}
$$

with

$$
\tilde{Q}_{p}^{(j)}=\sum_{m=0}^{\gamma-1}\left(T_{p}-\tilde{B}_{p}^{(j)} \tilde{A}_{p}\right)^{m} \tilde{B}_{p}^{(j)}, \quad j=3,4,
$$

for some positive integer $\gamma$.

The operators $\tilde{B}_{p}^{(j)}, j=1,2,3,4$, are all mappings on $X$ into $X_{p}$. The solution $\tilde{f}_{p} \in X_{p}$ of (4.1) is approximated by a defect correction process of the form 


$$
\left\{\begin{array}{l}
\tilde{f}_{p, 0}=0, \\
\tilde{f}_{p, i+1}=\tilde{B}_{p} g_{p}+\left(I-\tilde{B}_{p} \tilde{A}_{p}\right) \tilde{f}_{p, i} .
\end{array}\right.
$$

We notice that $\tilde{B}_{p}^{(1)}$ and $\tilde{B}_{p}^{(3)}$ yield iterative processes that are equivalent, respectively, with the "One Step Method" and the "Multi Grid Method" discussed in Hackbusch [7]. $\tilde{B}_{p}^{(2)}$ yields an iterative process analogous to Atkinson's method, whereas $\tilde{B}_{p}^{(4)}$ yields a new multiple grid method with better convergence properties than $\tilde{B}_{p}^{(3)}$.

Analogously to Section 3, but restricting the domain of the operators to $X_{p}$, we see that here $\tilde{Q}_{p}^{(j)}: X_{p} \rightarrow X_{p}$ is an approximate inverse of $\tilde{A}_{p}: X_{p} \rightarrow X_{p}$, and the amplification operator on $X_{p}$ into $X_{p}$ of a defect correction step with $\tilde{Q}_{p}^{(j)}$ is

$$
T_{p}-\tilde{Q}_{p}^{(j)} \tilde{A}_{p}=\left(T_{p}-\tilde{B}_{p}^{(j)} \tilde{A}_{p}\right)^{\gamma} .
$$

Thus, one application of $\tilde{Q}_{p}^{(j)}$ is equivalent to the $\gamma$ times application of $\tilde{B}_{p}^{(j)}$, and we may write

$$
\tilde{Q}_{p}^{(j)}=\left[T_{p}-\left(T_{p}-\tilde{B}_{p}^{(j)} \tilde{A}_{p}\right)^{\gamma}\right] \tilde{A}_{p}^{-1} .
$$

The convergence of the process (4.2) depends on the Lipschitz constant of the operator $I-\tilde{B}_{p} \tilde{A}_{p}$ as a mapping $X_{p} \rightarrow X_{p}$. Therefore its rate of convergence is given by $\left\|T_{p}\left(I-\tilde{B}_{p} \tilde{A}_{p}\right)\right\|$. This rate of convergence is studied in the remainder of this section.

TheOREM 4.1. (i) $\left\|T_{p}\left(I-\tilde{B}_{p}^{(1)} \tilde{A}_{p}\right)\right\| \rightarrow 0$ as $p \rightarrow \infty$,

(ii) $\left\|T_{p}\left(I-\tilde{B}_{p}^{(2)} \tilde{A}_{p}\right)\right\| \leqslant \tilde{C}\left(X_{0}\right)$ as $p \rightarrow \infty, \tilde{C}\left(X_{0}\right)<1$ for $X_{0}$ sufficiently large.

Proof. (i) Substitution of the explicit expressions for $\tilde{B}_{p}^{(1)}$ and $\tilde{A}_{p}$ yields

$$
\begin{aligned}
T_{p}\left(I-\tilde{B}_{p}^{(1)} \tilde{A}_{p}\right)= & \left(T_{p}-T_{p-1}\right) K_{p} \\
& +T_{p-1}\left(I-T_{p-1} K_{p-1}\right)^{-1} T_{p}\left(K_{p}-T_{p-1} K_{p-1}\right) K_{p} .
\end{aligned}
$$

Therefore, we have

$$
\begin{aligned}
\left\|T_{p}\left(I-\tilde{B}_{p}^{(1)} \tilde{A}_{p}\right)\right\| \leqslant & \left\|\left(I-T_{p}\right) K_{p}\right\|+\left\|\left(I-T_{p-1}\right) K_{p}\right\| \\
& +\left\|T_{p-1}\right\|\left\|\left(I-T_{p-1} K_{p-1}\right)^{-1}\right\|\left\|T_{p}\right\| \\
& \cdot\left\{\left\|\left(K_{p}-K\right) K_{p}\right\|+\left\|\left(K-K_{p-1}\right) K_{p}\right\|\right. \\
& \left.\quad+\left\|\left(K_{p-1}-T_{p-1} K_{p-1}\right) K_{p}\right\|\right\} .
\end{aligned}
$$

Using Lemmas 2.1 to 2.5 we obtain the proof of (i) by the same arguments as used for the proof of Theorem 3.1.

(ii) Replacing the subscript $p-1$ by 0 in the first part of the proof, we get

$$
\begin{gathered}
\left\|T_{p}\left(I-\tilde{B}_{p}^{(2)} \tilde{A}_{p}\right)\right\| \leqslant\left\|\left(I-T_{p}\right) K_{p}\right\|+\left\|\left(I-T_{0}\right) K_{p}\right\|+\left\|T_{0}\right\|\left\|\left(I-T_{0} K_{0}\right)^{-1}\right\|\left\|T_{p}\right\| \\
\cdot\left\{\left\|\left(K_{p}-K\right) K_{p}\right\|+\left\|\left(K-K_{0}\right) K_{p}\right\|+\left\|\left(I-T_{0}\right) K_{0}\right\|\left\|K_{p}\right\|\right\} .
\end{gathered}
$$

For $p \rightarrow \infty\left\|\left(I-T_{p}\right) K_{p}\right\|$ and $\left\|\left(K_{p}-K\right) K_{p}\right\|$ vanish, whereas the other terms tend to a constant value depending on $X_{0}$.

Definition.

$$
\eta_{p}^{(j)}=\left\|T_{p}\left(I-\tilde{B}_{p}^{(j)} \tilde{A}_{p}\right)\right\|, \quad j=1,2,3,4
$$


THEOREM 4.2.

$$
\begin{aligned}
& \eta_{p}^{(3)}<\eta_{p}^{(1)}+\eta_{p-1}^{(3)^{\gamma}}\left[\eta_{p}^{(1)}+\left\|T_{p}\right\|\left\|K_{p}\right\|\right] \\
& \eta_{p}^{(4)}<\eta_{p}^{(1)}+\eta_{p-1}^{(4)^{\gamma}}\left[\eta_{p}^{(1)}+\left\|T_{p}\right\|\right] .
\end{aligned}
$$

Proof. We use the notation $M_{R}^{(j)}=T_{p}\left(I-\tilde{B}_{p}^{(j)} \tilde{A}_{p}\right), j=1,3,4$. From assumption (A6) and the definitions of $A_{p}, \tilde{A}_{p}$, and $\tilde{B}_{p}^{(j)}, j=1,3,4$, it is clear that

$$
T_{p} A_{p}=T_{p} \tilde{A}_{p}=\tilde{A}_{p} T_{p}
$$

and

$$
\tilde{B}_{p}^{(j)}=T_{p} \tilde{B}_{p}^{(j)}=\tilde{B}_{p}^{(j)} T_{p}, \quad j=1,3,4
$$

Hence,

$$
M_{p}^{(j)}=T_{p} M_{p}^{(j)}=M_{p}^{(j)} T_{p}, \quad j=1,3,4
$$

and also

$$
\tilde{Q}_{p}^{(j)}=\left[T_{p}-M_{p}^{(j)^{\gamma}}\right] \tilde{A}_{p}^{-1}
$$

(i) From the definition of $\tilde{B}_{p}^{(1)}$, we get

$$
M_{p}^{(1)}=T_{p}-T_{p} \tilde{A}_{p}-T_{p-1} \tilde{A}_{p-1}^{-1} T_{p} K_{p} \tilde{A}_{p}=T_{p} K_{p}-T_{p-1} \tilde{A}_{p-1}^{-1} T_{p} K_{p} \tilde{A}_{p} .
$$

These relations are used to prove that

$$
\begin{aligned}
M_{p}^{(3)} & =T_{p}\left[I-\left\{T_{p}+T_{p-1}\left(T_{p-1}-M_{p}^{(3)^{\gamma}}\right) \tilde{A}_{p-1}^{-1} T_{p} K_{p}\right\} \tilde{A}_{p}\right] \\
& =T_{p}-T_{p} \tilde{A}_{p}-T_{p-1}\left(T_{p-1}-M_{p-1}^{(3)}\right) \tilde{A}_{p-1}^{-1} T_{p} K_{p} \tilde{A}_{p} \\
& =M_{p}^{(1)}+M_{p-1}^{(3)^{\gamma}} T_{p-1} \tilde{A}_{p-1}^{-1} T_{p} K_{p} \tilde{A}_{p} \\
& =M_{p}^{(1)}+M_{p-1}^{(3)^{\gamma}}\left(T_{p} K_{p}-M_{p}^{(1)}\right) .
\end{aligned}
$$

Hence,

$$
\eta_{p}^{(3)} \leqslant \eta_{p}^{(1)}+\eta_{p-1}^{(3)}\left(\eta_{p}^{(1)}+\left\|T_{p}\right\|\left\|K_{p}\right\|\right)
$$

(ii)

$$
\begin{aligned}
M_{p}^{(4)} & =T_{p}-T_{p} \tilde{A}_{p}+T_{p-1} \tilde{A}_{p}-\left(T_{p-1}-M_{p-1}^{(4)}\right) \tilde{A}_{p-1}^{-1}\left(T_{p-1} \tilde{A}_{p-1}+T_{p} K_{p}\right) \tilde{A}_{p} \\
& =M_{p}^{(1)}+M_{p-1}^{(4)} \tilde{A}_{p-1}^{-1}\left(\tilde{A}_{p-1} T_{p-1}+T_{p} K_{p}\right) \tilde{A}_{p} \\
& =M_{p}^{(1)}+M_{p-1}^{(4)} T_{p-1} T_{p}\left(\tilde{A}_{p}+\tilde{A}_{p-1}^{-1} T_{p} K_{p} \tilde{A}_{p}\right) \\
& =M_{p}^{(1)}+M_{p-1}^{(4)}\left(T_{p}-M_{p}^{(1)}\right) .
\end{aligned}
$$

Hence,

$$
\eta_{p}^{(4)} \leqslant \eta_{p}^{(1)}+\eta_{p-1}^{(4)^{\gamma}}\left(\eta_{p}^{(1)}+\left\|T_{p}\right\|\right) \text {. Q.E.D. }
$$

THEOREM 4.3. Let $\gamma \geqslant 2$ and let $\eta_{p}^{(1)}$ satisfy

$$
\eta_{p}^{(1)} \leqslant v_{p}=d^{p} v_{0} \quad \text { for some } 0<d<1 \text {. }
$$

Then,

(i) if $v_{0} \leqslant\left\{\sqrt{d^{2}+C_{1}^{2} C_{2}^{2}}-C_{1} C_{2}\right\} / 2 d$, it follows that $\eta_{p}^{(3)} \leqslant 2 v_{0} d^{p}$, and

(ii) if $v_{0} \leqslant\left\{\sqrt{d^{2}+C_{1}^{2}}-C_{1}\right\} / 2 d$, it follows that $\eta_{p}^{(4)} \leqslant 2 v_{0} d^{p}$. 
Proof. (i) Use Lemma 3.3 with $k=C_{1} C_{2}$ and Theorem 4.2.

(ii) Analogously with $k=C_{1}$.

By $\tilde{f}_{p, \sigma}^{(j)}$ we denote the result of $\sigma$ applications of the Defect Correction Process on level $p$ with approximate inverse $\tilde{B}_{p}^{(j)}, j=1,3,4$, when we take zero as the initial approximate.

THEOREM 4.4 [APPROXIMATION THEOREM]. Under the hypotheses of Theorem 4.3 the Multi Grid process yields $\tilde{f}_{p, \sigma}^{(j)}$, for which the following error estimates hold

$$
\left\|f-\tilde{f}_{p, \boldsymbol{\sigma}}^{(j)}\right\| \leqslant\left\|f-T_{p} f\right\|+C_{1} C_{4}\left\|K f-K_{p} f\right\|+\left(2 d^{p} v_{0}\right)^{\sigma}\left\|\tilde{f}_{p}\right\|,
$$

where $f$ and $\tilde{f}_{p}$ are the solutions of (2.1) and (2.3) respectively.

Proof. For $j=1,3,4$ we have

$$
\left\|f-\tilde{f}_{p, \boldsymbol{\sigma}}^{(j)}\right\| \leqslant\left\|f-T_{p} f\right\|+\left\|T_{p} f-\tilde{f}_{p}\right\|+\left\|\tilde{f}_{p}-\tilde{f}_{\boldsymbol{p}, \boldsymbol{\sigma}}^{(j)}\right\|
$$

and the proof follows from Lemma 2.6 and Theorem 4.3.

We notice that the usual discretization methods easily satisfy the first condition of Theorem 4.3 as is illustrated in Section 5. The other condition of Theorem 4.3, which requires an upper bound on $v_{0}$, essentially is a requirement on the coarsest discretization used in the multiple grid algorithm. This condition is also discussed in the next section.

5. Numerical Results. In this section we illustrate the theoretical convergence results from the previous sections, and we make some remarks about the computational complexity of the various methods. We shall only show numerical results obtained with the methods that appear to be the most efficient. These methods are defined by the approximate inverses $B_{p}^{(2)}$ (Atkinson's method), $\tilde{B}_{p}^{(3)}$ (Hackbusch's method) and $\tilde{B}_{p}^{(4)}$ (a new method with better convergence properties).

As an example, the integral equation

$$
f(x)-\lambda \int_{0}^{1} \cos (\pi x y) f(y)=g(x)
$$

is solved for various values of the parameter $\lambda$ (cf. Hackbusch [7] who gives results for the same equation); $g(x)$ is chosen such that $f(x)=e^{x} \cos (7 x)$.

The operators $K_{p}$ are defined by means of the repeated trapezoidal rule:

$$
K_{p} f(x)=\sum_{j=0}^{N_{p}} w_{j} k\left(x, x_{j}\right) f\left(x_{j}\right),
$$

where the nodal points $\left\{x_{j}\right\}$ are uniformly distributed $\left(x_{0}=0, x_{N_{p}}=1\right)$ and the weights $\left\{w_{j}\right\}$ are given by $\left\{\frac{1}{2} h_{p}, h_{p}, h_{p}, \ldots, h_{p}, \frac{1}{2} h_{p}\right\}$ with $h_{p}=\left(N_{p}\right)^{-1}$. The projection operators are defined by piecewise linear interpolation at the nodal points $\left\{x_{j}\right\}$. The different grid-levels are related by $N_{p}=2 N_{p-1}$.

For the operators $\left\{K_{p}\right\}$ and $\left\{T_{p}\right\}$ we know (cf. Atkinson [3] and Prenter [9])

$$
\begin{aligned}
& \left\|K_{p} f-K f\right\|=\vartheta\left(h_{p}^{2}\right), \\
& \left\|T_{p} f-f\right\|=\vartheta\left(h_{p}^{2}\right), \\
& a_{p}=\vartheta\left(h_{p}^{2}\right), \quad \text { and } \\
& b_{p}=\vartheta\left(h_{p}^{2}\right) \text { for } p \rightarrow \infty .
\end{aligned}
$$


Using these estimates, we easily derive (see the proof of Theorem 4.1)

$$
\eta_{p}^{(1)} \leqslant v_{p}=C h_{p-1}^{2} \text {. }
$$

Because the successive meshsizes are related by $h_{p}=h_{0} 2^{-p}$, we have

$$
\eta_{p}^{(1)} \leqslant v_{p}=4 C h_{0}^{2} 4^{-p} \text {. }
$$

Comparing this expression with the assumption on $\eta_{p}^{(1)}$ in Theorem 4.3 , we see that $d=1 / 4$. In the same theorem, conditions on $\eta_{0}^{(1)}$ are formulated for the multiple grid methods to converge. Comparing these conditions, we conclude that the condition on $\eta_{0}^{(1)}$ in the process defined by $\tilde{B}_{p}^{(4)}$ is independent of $C_{2}=$ $\sup _{p>0}\left\|K_{p}\right\|$, whereas in the process defined by $\tilde{B}_{p}^{(3)}$ the condition on $\eta_{0}^{(1)}$ becomes stronger as $\sup _{p>0}\left\|K_{p}\right\|$ increases. In Figure 1 we sketch the regions of convergence induced by $\tilde{B}_{p}^{(3)}$ and $\tilde{B}_{p}^{(4)}$, as derived from Theorem 4.3 with $d=1 / 4$ and $\gamma=2$.
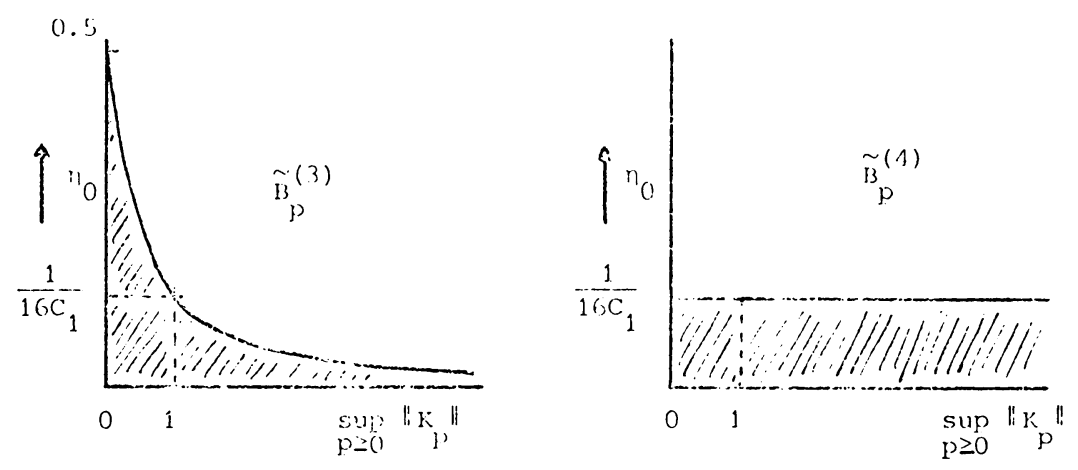

FIGURE 1

The multiple grid convergence regions. The coarsest grid convergence factor $\eta_{0}$ versus $C_{2}=\sup _{p>0}\left\|K_{p}\right\|$.

Hence, from Theorem 4.3 one may expect that both multiple grid methods yield similar results as $\|K\| \approx 1$, whereas they differ for $\|K\| \gg 1$. For the integral equation (5.1), $\|K\| \gg 1$ holds for $\lambda \gg 1$.

In Tables 5.1-5.3 we give the observed rates of convergence

$$
\eta\left(N_{p} ; N_{0}\right)=\left[\left\|\tilde{f}_{p, i+1}-\tilde{f}_{p, i}\right\| /\left\|\tilde{f}_{p, 1}-\tilde{f}_{p, 0}\right\|\right]^{1 / i},
$$

for the iterative methods defined by $B_{p}^{(2)}, \tilde{B}_{p}^{(3)}$ and $\tilde{B}_{p}^{(4)}$, respectively, with $\gamma=2$. The dependence of $\eta\left(N_{p} ; N_{0}\right)$ on $N_{p}$, the number of mesh intervals in the finest grid, and on $N_{0}$, the number in the coarsest grid, is shown. The value of $i$ is suitably chosen and $\|\cdot\|$ denotes the maximum norm. From Table 5.1 we see that the rates of convergence of Atkinson's method tend to a constant value as $N_{p} \rightarrow \infty$. As was expected, it decreases as $N_{0}$ increases.

In the case of convergence, the Tables 5.2 and 5.3 asymptotically show similar results. However, for larger values of $\lambda$, the new multiple grid method needs fewer subintervals in the coarsest grid. The quotients $\eta\left(N_{p} ; N_{0}\right) / \eta\left(N_{p-1} ; N_{0}\right)$ approximate the value $d=1 / 4$, which is in agreement with the theory. 
TABLE 5.1

Rates of convergence for the two-grid method defined by $B_{p}^{(2)}$ (Atkinson's method).

\begin{tabular}{|c|c|c|c|c|c|c|c|}
\hline$\lambda$ & $\mathrm{N}_{\mathrm{p}}$ & 2 & 4 & 8 & 16 & 32 & 64 \\
\hline 1 & $\begin{array}{r}4 \\
8 \\
16 \\
32 \\
64 \\
128\end{array}$ & $\begin{array}{l}.2310^{-1} \\
.2810^{-1} \\
.3010^{-1} \\
.3010^{-1} \\
.3010^{-1} \\
.3010^{-1}\end{array}$ & $\begin{array}{l}.5810^{-2} \\
.7210^{-2} \\
.7610^{-2} \\
.7810^{-2} \\
.7810^{-2}\end{array}$ & $\begin{array}{l}.1510^{-2} \\
.1310^{-2} \\
.1910^{-2} \\
.1910^{-2}\end{array}$ & $\begin{array}{l}.3810^{-3} \\
.4710^{-3} \\
.5210^{-3}\end{array}$ & $\begin{array}{l}.8310^{--4} \\
.1010^{-3}\end{array}$ & $.2410^{-4}$ \\
\hline 10 & $\begin{array}{r}4 \\
8 \\
16 \\
32 \\
64 \\
123\end{array}$ & $\begin{array}{l}.1110^{+1} \\
.1610^{+1} \\
.1710^{+1} \\
.1710^{+1} \\
.1710^{+1} \\
.1710^{+1}\end{array}$ & $\begin{array}{l}.1810^{\circ} \\
.2210^{0} \\
.2310^{\circ} \\
.2410^{\circ} \\
.2410^{0}\end{array}$ & $\begin{array}{l}.3610^{-1} \\
.4510^{-1} \\
.4810^{-1} \\
.4810^{-1}\end{array}$ & $\begin{array}{l}.8610^{-2} \\
.1110^{-1} \\
.1110^{-1}\end{array}$ & $\begin{array}{l}.2110^{-2} \\
.2710^{-2}\end{array}$ & $.3810^{-3}$ \\
\hline 100 & $\begin{array}{r}4 \\
8 \\
16 \\
32 \\
64 \\
128\end{array}$ & $\begin{array}{l}.6410^{+1} \\
.1110^{+2} \\
.1410^{+2} \\
.1510^{+2} \\
.1510^{+2} \\
.1510^{+2}\end{array}$ & $\begin{array}{l}.1410^{+1} \\
.1610^{+1} \\
.1610^{+1} \\
.1610^{+1} \\
.1610^{+1}\end{array}$ & $\begin{array}{l}.4010^{0} \\
.4210^{0} \\
.4510^{0} \\
.4910^{0}\end{array}$ & $\begin{array}{l}.9910^{-1} \\
.1510^{0} \\
.1610^{0}\end{array}$ & $\begin{array}{l}.3310^{-1} \\
.4110^{-1}\end{array}$ & $.6810^{-2}$ \\
\hline
\end{tabular}

Using (5.2), (5.3), and (5.6) for the approximation errors, we conclude, from Theorem 4.4, that for the multiple grid methods $\sigma=2$ iteration steps are sufficient to get an iteration error which is of the same order of magnitude as the approximation errors $\left\|f-T_{p} f\right\|$ and $\left\|K f-K_{p} f\right\|$. Of course, this is not the case with Atkinson's method for which one has to perform $\Theta\left(\log N_{p}\right)$ iteration steps. That these asymptotic arguments hold already for relatively small $N_{p}$ is shown in the Tables 5.4-5.5, where we compare the approximation errors with the iteration error after $\sigma=2$ iteration steps.

We conclude this section with some remarks about the asymptotic computational complexity. We only count multiplications, ignoring the multiplications with the weights $w_{j}$ and the computations involved in the evaluation of $k(s, t)$. Then, asymptotically for $N_{p} \rightarrow \infty$, the operation counts per iteration sweep for the various approximate inverses are:

$$
\begin{array}{llll}
B_{p}^{(1)}: & 2.75 N_{p}^{2}, & \tilde{B}_{p}^{(1)}: 1.75 N_{p}^{2}, \\
B_{p}^{(2)}: & 2 N_{p}^{2}, & \tilde{B}_{p}^{(2)}: 1 N_{p}^{2}, \\
B_{p}^{(3)}: & 2 N_{p}^{2}{ }^{2} \log N_{p}, & \tilde{B}_{p}^{(3)}: & 3 N_{p}^{2}, \\
B_{p}^{(4)}: & 2.5 N_{p}^{2}{ }^{2} \log N_{p}, & \tilde{B}_{p}^{(4)}: & 3.5 N_{p}^{2} .
\end{array}
$$


TABLE 5.2

Rates of convergence for the multiple grid method defined by $\tilde{B}_{p}^{(3)}$ (Hackbusch's method).

\begin{tabular}{|c|c|c|c|c|c|c|c|}
\hline$\lambda$ & $N_{p}$ & 2 & 4 & 8 & 16 & 32 & 64 \\
\hline 1 & $\begin{array}{r}4 \\
8 \\
16 \\
32 \\
64 \\
128\end{array}$ & $\begin{array}{l}.3110^{-1} \\
.0810^{-2} \\
.2110^{-2} \\
.6210^{-3} \\
.1110^{-3} \\
.3510^{-4}\end{array}$ & $\begin{array}{l}.9410^{-2} \\
.2410^{-2} \\
.6210^{-3} \\
.1410^{-3} \\
.3510^{-4}\end{array}$ & $\begin{array}{l}.2310^{-2} \\
.6210^{-3} \\
.1410^{-3} \\
.3510^{-4}\end{array}$ & $\begin{array}{l}.6210^{-3} \\
.1410^{-3} \\
.3510^{-4}\end{array}$ & $\begin{array}{l}.1410^{-3} \\
.3410^{-4}\end{array}$ & $.3510^{-4}$ \\
\hline 10 & $\begin{array}{r}4 \\
8 \\
16 \\
32 \\
61 \\
128\end{array}$ & $\begin{array}{l}.3210^{0} \\
.1210^{+1} \\
.4210^{+1} \\
.2010^{+3} \\
.2310^{+6} \\
.4010^{+12}\end{array}$ & $\begin{array}{l}.1010^{0} \\
.1210^{0} \\
.1810^{-1} \\
.9110^{-2} \\
.4610^{-3}\end{array}$ & $\begin{array}{l}.2510^{-1} \\
.1310^{-1} \\
.2410^{-2} \\
.5710^{-3}\end{array}$ & $\begin{array}{l}.6210^{-2} \\
.2310^{-2} \\
.5210^{-3}\end{array}$ & $\begin{array}{l}.1910^{-2} \\
.5310^{-3}\end{array}$ & $.5110^{-3}$ \\
\hline 100 & $\begin{array}{r}4 \\
8 \\
16 \\
32 \\
64 \\
128\end{array}$ & $\begin{array}{l}.4310^{+1} \\
.1110^{+4} \\
.6610^{+7} \\
.1710^{+17} \\
.8210^{+34} \\
.8010^{+70}\end{array}$ & $\begin{array}{l}.1110^{+1} \\
.7710^{+2} \\
.7910^{+4} \\
.4610^{+11} \\
.8610^{+23}\end{array}$ & $\begin{array}{l}.2910^{0} \\
.5110^{+1} \\
.1510^{+3} \\
.9610^{+7}\end{array}$ & $\begin{array}{l}.1010^{0} \\
.3310^{0} \\
.4310^{\circ}\end{array}$ & $\begin{array}{l}.2910^{-1} \\
.2410^{-1}\end{array}$ & $.8510^{-2}$ \\
\hline
\end{tabular}

Here we ignored the direct solution on the coarsest grid and we applied the multiple grid methods with $\sigma=\gamma=2$ on all levels.

Note. The number of kernel-function evaluations is $N_{p}^{2}$ in the linear case when they are computed once and stored. In the nonlinear case or in the case when kernel-functions are reevaluated whenever they are used, the number of kernelfunction evaluations is of the same order as the number of arithmetic operations.

Asymptotically, all methods need only 2 iterations to obtain a result of the order of the truncation error, except the methods with $B_{p}^{(2)}$ and $\tilde{B}_{p}^{(2)}$ which need $\Theta\left(\log N_{p}\right)$ sweeps. For the methods with $B_{p}^{(1)}$ and $\tilde{B}_{p}^{(1)}$ the coarsest grid still has $N_{p} / 2$ mesh intervals; on this grid the problem is solved by a direct method (e.g. Gauss-elimination) and therefore we have to add $\frac{1}{12} N_{p}^{3}$ to the total computational complexity. Thus, for the total amount of asymptotic computational work we get the following table:

$$
\begin{aligned}
& B_{p}^{(1)}: \frac{1}{12} N_{p}^{3}+5.5 N_{p}^{2}, \quad \tilde{B}_{p}^{(1)}: \frac{1}{12} N_{p}^{3}+3.5 N_{p}^{2}, \\
& B_{p}^{(2)}: \quad \frac{2}{3} N_{0}^{3}+\mathcal{O}\left(N_{p}^{2} \log N_{p}\right), \quad \tilde{B}_{p}^{(2)}: \quad \frac{2}{3} N_{0}^{3}+\mathcal{O}\left(N_{p}^{2} \log N_{p}\right),
\end{aligned}
$$




$$
\begin{aligned}
& B_{p}^{(3)}: \quad \frac{2}{3} N_{0}^{3}+4 N_{p}^{2}{ }^{2} \log N_{p}, \quad \tilde{B}_{p}^{(3)}: \frac{2}{3} N_{0}^{3}+6 N_{p}^{2}, \\
& B_{p}^{(4)}: \frac{2}{3} N_{0}^{3}+5 N_{p}^{2}{ }^{2} \log N_{p}, \quad \tilde{B}_{p}^{(4)}: \frac{2}{3} N_{0}^{3}+7 N_{p}^{2} \text {. }
\end{aligned}
$$

TABLE 5.3

\begin{tabular}{|c|c|c|c|c|c|c|c|}
\hline$\lambda$ & $N_{p}^{N_{0}}$ & 2 & 4 & 8 & 16 & 32 & 64 \\
\hline \multirow{6}{*}{1} & 4 & $.3110^{-1}$ & & & & \multirow[b]{5}{*}{$.1410^{-3}$} & \multirow[b]{6}{*}{$.3510^{-4}$} \\
\hline & 8 & $.9510^{-2}$ & $.9410^{-2}$ & & & & \\
\hline & 16 & $.2310^{-2}$ & $.2310^{-2}$ & $.2310^{-2}$ & & & \\
\hline & 32 & $.6210^{-3}$ & $.6210^{-3}$ & $.6210^{-3}$ & $.6210^{-3}$ & & \\
\hline & 64 & $.1410^{-3}$ & $.1410^{-3}$ & $.1410^{-3}$ & $.14 \div 0^{-3}$ & & \\
\hline & 128 & $.3510^{-4}$ & $.3410^{-4}$ & $.3410^{-4}$ & $.3510^{-4}$ & $.3510^{-4}$ & \\
\hline \multirow{6}{*}{10} & 4 & $.3210^{0}$ & & & & \multirow{6}{*}{$.1910^{-2}$} & \multirow[b]{6}{*}{$.5110^{-3}$} \\
\hline & 8 & $.1810^{0}$ & $.1010^{0}$ & & & & \\
\hline & 16 & $.4010^{-1}$ & $.1210^{-1}$ & $.2510^{-1}$ & & & \\
\hline & 32 & $.7010^{-2}$ & $.6910^{-2}$ & $.6010^{-2}$ & $.6210^{-2}$ & & \\
\hline & 64 & $.1910^{-2}$ & $.1910^{-2}$ & $.1910^{-2}$ & $.1910^{-2}$ & & \\
\hline & 128 & $.5010^{-3}$ & $.5010^{-3}$ & $.5010^{-3}$ & $.5010^{-3}$ & & \\
\hline \multirow{6}{*}{100} & 4 & $.4310^{+1}$ & & & & \multirow{6}{*}{$.2910^{-1}$} & \multirow[b]{6}{*}{$.8510^{-2}$} \\
\hline & 8 & $.7210^{+1}$ & $.1110^{+1}$ & & & & \\
\hline & 16 & $.3010^{+2}$ & $.1110^{+1}$ & $.2910^{0}$ & & & \\
\hline & 32 & $.1310^{+4}$ & $.1310^{+1}$ & $.2010^{0}$ & $.1010^{\circ}$ & & \\
\hline & 64 & $.1710^{+7}$ & $.1610^{+1}$ & $.4110^{-1}$ & $.3610^{-1}$ & & \\
\hline & 128 & $.2910^{+13}$ & $.2610^{+1}$ & $.7510^{-2}$ & $.9410^{-2}$ & & \\
\hline
\end{tabular}

Rates of convergence for the multiple grid method defined by $\tilde{B}_{p}^{(4)}$.

From these tables we see that the multiple grid methods become cheaper than Atkinson's method, whenever the latter needs more than three iterations.

In order to get an impression of the qualities of the various methods, we suggest to measure by experiments the following ratio (which shows the amount of computational work per digit accuracy obtained):

$$
\kappa_{\sigma}=-\frac{\text { Number of multiplications to obtain } \tilde{f}_{p, \sigma}}{N_{p}^{2}{ }^{10} \log \left\|f-\tilde{f}_{p, \sigma}\right\|}
$$

Because of their rates of convergence, for the multiple grid methods we choose $\sigma=2$, whereas for Atkinson's method we determine $\sigma$ such that $\kappa_{\sigma}$ is minimal. Better methods are now characterized by a smaller $\kappa_{\boldsymbol{\sigma}}$. 
TABLE 5.4

The ratio: iteration error after 2 sweeps / approximation errors, i.e.

$$
\left\|\tilde{f}_{p, 2}-\tilde{f}_{p, \infty}\right\| /\left\|f-\tilde{f}_{p, \infty}\right\|,
$$

Numbers of subintervals: $N_{0}=2$, ( $a$ divergent iteration process is denoted by -).

\begin{tabular}{|c|r|ccc|}
\hline$\lambda$ & $N_{p}$ & $\mathrm{~B}_{\mathrm{p}}^{(2)}$ & $\tilde{B}_{\mathrm{p}}^{(3)}$ & $\tilde{B}_{\mathrm{p}}^{(4)}$ \\
\hline 1 & 4 & .0046 & .0018 & .0018 \\
& 8 & .0267 & .0003 & .0003 \\
& 16 & .1162 & .0001 & .0001 \\
& 32 & .4743 & .0000 & .0000 \\
& 64 & .0930 & .0000 & .0000 \\
& 128 & 5.6378 & .0000 & .0000 \\
\hline \multirow{1}{*}{10} & 4 & - & 3.3089 & 3.3089 \\
& 8 & - & - & .0508 \\
& 16 & - & - & .3899 \\
& 32 & - & - & .0694 \\
& 64 & - & - & .0194 \\
& 128 & - & - & .0050 \\
\hline
\end{tabular}

TABLE 5.5

The ratio: iteration error after 2 sweeps / approximation errors.

As Table 5.4, but $N_{0}=8$.

\begin{tabular}{|c|c|ccc|}
\hline$\lambda$ & $\mathrm{N}_{\mathrm{p}}$ & $\mathrm{B}_{\mathrm{p}}^{(2)}$ & $\tilde{B}_{\mathrm{p}}^{(3)}$ & $\tilde{\mathrm{B}}_{\mathrm{p}}^{(4)}$ \\
\hline 1 & 16 & .0003 & .0001 & .0001 \\
& 32 & .0017 & .0000 & .0000 \\
& 64 & .0075 & .0000 & .0000 \\
& 128 & .0306 & .0000 & .0000 \\
\hline 10 & 16 & .0936 & .3202 & .3202 \\
& 32 & .6088 & .2000 & .0692 \\
& 64 & 2.7111 & .0341 & .0194 \\
& 128 & 11.1310 & .0056 & .0050 \\
\hline 100 & 16 & 91.0760 & 34.0160 & 34.0160 \\
& 32 & 563.4392 & - & 34.7089 \\
& 64 & 2480.5082 & - & 24.3138 \\
& 128 & $>10^{4}$ & - & 0.1220 \\
\hline
\end{tabular}


TABLE 5.6

For problem (5.1) with $\lambda=100$ and $N_{p}=128$ the experimental ratios $\kappa_{\sigma}$, where $\sigma$ is given between parentheses;

for this problem ${ }^{10} \log \left\|f-\tilde{f}_{p, \infty}\right\|=-3.5$.

\begin{tabular}{|c|c|c|c|c|}
\hline $\mathrm{N}_{0}$ & 8 & 16 & 32 & 64 \\
\hline $\mathrm{B}_{\mathrm{p}}^{(2)}$ & 7.55 (11) & $3.46(5)$ & $2.73 \quad(3)$ & $6.20 \quad(2)$ \\
\hline$\widetilde{B}_{p}^{(3)}$ & - & -8.42 (2) & $2.97 \quad(2)$ & $5.95 \quad(2)$ \\
\hline$\tilde{B}_{\mathrm{p}}^{(4)}$ & $2.00 \quad(2)$ & $1.86 \quad(2)$ & $2.20 \quad(2)$ & $6.10 \quad(2)$ \\
\hline
\end{tabular}

Table 5.6 shows, for the multiple grid methods, that small values of $N_{0}$ are more efficient as long as the process converges. However, within a reasonable range of small $N_{0}$, it seems not worthwhile to determine an optimal $N_{0}$.

The asymptotic work estimates and the convergence property discussed in Section 4 lead us to prefer $\tilde{B}_{p}^{(3)}$ for $\|K\| \approx 1$ and $\tilde{B}_{p}^{(4)}$ for $\|K\| \gg 1$. Finally, we remark that the same multiple grid techniques can be applied to nonlinear problems as well. Moreover, the structure of multiple grid algorithms yields estimates for the approximation and truncation errors in a natural way. All these features together can be used to construct an automatic program for solving Fredholm integral equations of the second kind. In fact, such a program has been constructed and some of the results have been reported in [10].

Department of Numerical Analysis

Stichting Mathematisch Centrum

1098 SJ Amsterdam, The Netherlands

1. P. M. Anselone, Collectively Compact Operator Approximation Theory, Prentice-Hall, Englewood Cliffs, N. J., 1971.

2. K. E. AtKinson, "Iterative variants of the Nystrom method for the numerical solution of integral equations," Numer. Math., v. 22, 1973, pp. 17-31.

3. K. E. AtKInson, A Survey of Numerical Methods for the Solution of Fredholm Integral Equations of the Second Kind, SIAM, Philadelphia, Pa., 1976.

4. H. BRAKHAGE, "Über die numerische Behandlung von Integralgleichungen nach der Quadraturformelmethode," Numer. Math., v. 2, 1960, pp. 183-196.

5. A. Brandt, "Multi-level adaptive solutions to boundary-value problems," Math. Comp., v. 31, 1977, pp. 333-390.

6. A. BRANDT, "Multi-level adaptive techniques for singular perturbation problems," in Numerical Analysis of Singular Perturbation Problems (P. W. Hemker and J. J. H. Miller, Eds.), Academic Press, London, 1979.

7. W. HaCKBUSCH, Die schnelle Auflösung der Fredholmschen Integralgleichung zweiter Art, Report 78-4, Universität zu Köln, 1978.

8. W. HАсквUSCH, An Error Analysis of the Nonlinear Multi-Grid Method of Second Kind, Report 78-15, Universität zu Köln, 1978.

9. P. M. PRENTER, "A collocation method for the numerical solution of integral equations," SIAM J. Numer. Anal., v. 10, 1973, pp. 570-581. 
10. H. SCHIPPERS, Multi-Grid Techniques for the Solution of Fredholm Integral Equations of the Second Kind, Colloq. Numerical Treatment of Integral Equations, MC-Syllabus 41, Mathematisch Centrum, Amsterdam, 1979.

11. H. J. STETrER, "The defect correction principle and discretization methods," Numer. Math., v. 29, 1978, pp. 425-443.

12. P. Wesseling \& P. SonNeVeld, "Numerical experiments with a multiple grid and preconditioned Lanczos type method," Proc. IUTAM-Symposium on Approximation Methods for Navier-Stokes Problems, Paderborn, Sept. 1979, Lecture Notes in Math., 771, Springer.

13. P. Wesselng, "The rate of convergence of a multiple grid method," to appear in: Proc. Dundee Biennial Conference on Numerical Analysis, June 1979, Lecture Notes in Math., Springer. (Available as Report NA-30, Delft University of Technology.) 